\title{
Treatment Outcome of Drug Resistance Tuberculosis From a Centre of Eastern Region, Nepal
}

\author{
Ghimire $H B,{ }^{1 *}$ Rimal $H S^{2}$ Parajuli $P^{3}$
}

\section{Affiliation:}

1. Lecturer, Department of Internal Medicine, Birat Medical College \& Teaching Hospital, Tankisinuwari-02, Morang, Nepal.

2. Associate Professor, Department of Paediatrics, Birat Medical College \& Teaching Hospital, Tankisinuwari-02, Morang, Nepal.

3. Matron, Birat Medical College \& Teaching Hospital, Tankisinuwari-02, Morang, Nepal.

\section{ARTICLE INFO}

Article History

Received : 07 Aug, 2016

Accepted : 06 Sept, 2016

Published : $20 \mathrm{Dec}, 2016$

(c) Authors retain copyright and grant the journal right of first publication with the work simultaneously licensed under Creative Commons Attribution License CC - BY 4.0 that allows others to share the work with an acknowledgement of the work's authorship and initial publication in this journal.

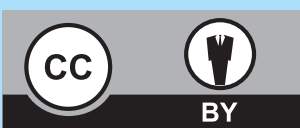

\section{* Corresponding Author}

Dr. Hridaya Bibhu Ghimire Lecturer

Department of Internal Medicine Birat Medical College \& Teaching Hospital

Tankisinuwari-02, Morang, Nepal

Email: hbghimire@hotmail.com

\section{Citation}

Ghimire HB, Rimal HS, Parajuli P. Treatment Outcome of Drug Resistance Tuberculosis From a Centre of Eastern Region, Nepal. BJHS 2016; 1 (1) 1:20-26.

\section{ABSTRACT}

\section{Introduction}

Drug resistance tuberculosis (DRTB) has become major problem worldwide with difficulty in treatment.

\section{Objective}

The study is conducted to find the incidence and outcomes of DRTB in one of the eastern tuberculosis treatment centre of Nepal.

\section{Methodology}

We collected all the cases of DRTB being treated from national anti-tuberculosis association of Biratnagar, Nepal. There were altogether 154 patients, who had sputum culture with resistance of at least one anti tubercular drug (ATD), included in this study.

\section{Results}

Of 154 total patients, 36 patients were resistant to rifampicin only, 84 patients were resistant to isoniazid and rifampicin, 18 patients were resistant to isoniazid, rifampicin and ethambutol and 16 patients were resistant to isoniazid, rifampicin and either streptomycin or fluoroquinolone. There was overall $71 \%$ cure rate in case of drug resistance tuberculosis. There was only statistical difference between cured and died patients in case of sputum conversion time with earlier sputum conversion in cured patients. Similarly, patients who were previously treated with category 2 ATD had only resistance to ethambutol or streptomycin or fluoroquinolone besides resistance to isoniazid and rifampicin compared to new patients and patients treated with category 1 regimen; as these groups were not found to be resistant to first three drugs.

\section{Conclusion}

There was overall $71 \%$ cure rate in case of drug resistance tuberculosis. Earlier sputum conversion was seen in cured patients compared to those who died during the treatment. Multiple drugs were resistant in patients previously treated with category 2 Anti-tubercular drugs.

\section{KEYWORDS}

Antituberculosis drugs, drug resistance tuberculosis, sputum conversion, treatment outcome 


\section{INTRODUCTION}

Globally it is estimated that $20 \%$ of previously treated tuberculosis (TB) cases and $3.3 \%$ of newly diagnosed TB cases are resistant to first line anti- tuberculosis drugs (ATD) or at least to isoniazid and rifampicin. ${ }^{1}$ These drug resistant tuberculosis (DRTB) strains have become a great threat for controlling TB from this world. ${ }^{2-3}$

Mono-resistance to single first line ATD is also being increasingly found. Reported prevalence of mono-resistance to isoniazid, one of the most important and potent first-line ATD, ranges from $4-12 \%$ for all TB cases and it is around $8 \%$ for new $\mathrm{TB}^{4-7}$ The prevalence of mono-resistance to rifampicin, another important first-line ATD, ranges from around $1-6 \%$ in different reports. ${ }^{6-9}$

Treatment of DRTB is somewhat difficult. Treatment of DRTB requires longer period with a combination of second-line drugs that are more expensive with greater toxicity than those used in standard first-line treatment regimens. In 2014 worldwide data showed that only $50 \%$ of multidrug resistant tuberculosis (MDRTB) cases were being successfully treated compared to $86 \%$ cure rate for newly diagnosed drug susceptible tuberculosis patients. ${ }^{1}$ Current recommendations for treatment of MDRTB require at least 20 months of ATD, ${ }^{10}$ although good success rates was seen in shorter regimen of therapy as well. ${ }^{11}$

Our study is conducted to find the outcomes of DRTB from national anti-tuberculosis association Biratnagar, one of the eastern tuberculosis treatment centres of Nepal.

\section{METHODOLOGY}

All the cases of DRTB, who started treatment from national anti-tuberculosis association of Biratnagar, Nepal from October 2005 with certain outcome till December 2014, were included in this study. Those patients who had sputum culture showing atypical mycobacteria were excluded. There were altogether 154 patients in whom sputum culture showed resistance to at least one drug (rifampicin) or multiple drugs (isoniazid+rifampicin or isoniazid+rifampicin+any other drugs) during the above mentioned study period in national anti-tuberculosis association of Biratnagar.

Among 154 DRTB patients, 137 of them were being previously treated with either category 1 or category 2 anti tuberculosis drugs. Because of treatment failure, sputum culture was done which showed the drug resistance patterns and were being designated as DRTB and treated for it. From December 2011, after the introduction of geneXpert technology for diagnosing tuberculosis cases, those patients who had positive sputum geneXpert test for mycobacterium tuberculosis and were resistant to rifampicin, were futher sent for sputum culture for confirming the presence of resistant bacteria and for identifying the resistant drugs. Xpert MTB/RIF (GeneXpert) is a newly-developed World Health Organization endorsed nucleic acid amplification test. This test detects Mycobacterium tuberculosis bacteria and it can also specify if there is any resistance to rifampicin drug in approximately two hours. ${ }^{12}$ This test is based on cartridge system requiring minimal training and biosafety measures ${ }^{13}$ and is being increasingly used for detecting Mycobacterium tuberculosis.

Sputum culture for mycobacterium tuberculosis was sent at GENETUP TB-laboratory. This laboratory functions as the National Reference Laboratory in the capital Kathmandu, Nepal and it uses the indirect proportion method on Löwenstein-Jensen medium for diagnosing the drug resistant tuberculosis. The external quality control for this laboratory using proportion method is done by the Supranational Laboratory in Gauting, Germany since 1994.

Laboratory follow-up was done for monitoring the treatment progress. Sputum smear microscopy and culture was done for this purpose and was conducted monthly during the first eight months of treatment and then every two months till the end of treatment.

All patients received the same standardized treatment regimen for drug resistant tuberculosis as according to national tuberculosis treatment protocol for drug resistance tuberculosis and in accordance with experts' opinion from the technical advisory committee. The regimen is composed of five drugs namely kanamycin, pyrazinamide, ethionamide, cycloserine and ofloxacin (later on ofloxacin was replaced by levofloxacinas according to international protocol, designed by centre for disease control and prevention (CDC), guidelines development group (GDG), which is also followed by national tuberculosis protocol of Nepal) in the intensive phase, which usually lasts eight months followed by all other drugs except kanamycin for the remaining time period (8Z-Km- OfxEto-Cs/16Z-Ofx-Eto-Cs).

Treatment outcome was categorized as cured, dead, failure and defaulted after 20 months of treatment to those patient enrolled after 2007 whereas after 24 months to those patient enrolled before 2007 (2005 to 2007 was a pilot phase program). Cured patients were defined as those patients who have clinical improvement of the symptoms followed by sputum smear and culture negative to mycobacterium tuberculosis by the eight month and end of the treatment. Those who died during the treatment period were designated as dead. Those whose sputum culture and smear were found to be positive after eight months of treatment were considered as failure of treatment. Those patients who lost follow up or had not completed the treatment due to variable reasons were the defaulted cases.

Data were entered and SPSS 16 was used for analyzing the data results. Cross tabs, Chi square test, non parametric test and individual sample $t$ test were used during the analysis. Any results with $p<0.05$ was considered to be statistically significant. 


\section{RESULTS}

154 DRTB patients were detected from sputum culture in this study, of which 109 patients (70.78\%) were cured completely, $26(16.88 \%)$ of them died, six patients $(3.90 \%)$ developed failure in treatment and 13 (8.44\%) of them defaulted from our centre as shown in Figure 1.

Figure 1: Drug resistant tuberculosis treatment outcome

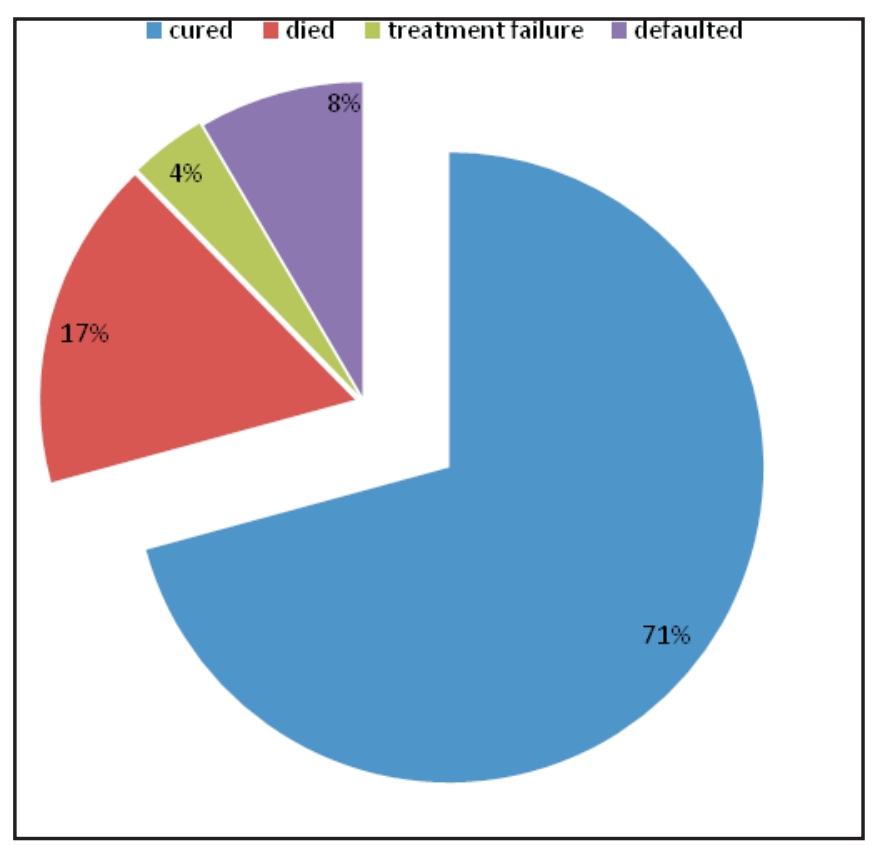

Among the total 154 DRTB patients, 36 patients were resistant to rifampicin followed by 84 resistant to isoniazid and rifampicin, 18 resistant to isoniazid, rifampicin and ethambutol and 16 resistant to isoniazid, rifampicin and either streptomycin or fluoroquinolone. Out of these 36 patients resistant to rifampicin, 25 patients were cured, seven died, two patients developed treatment failure and two of them were defaulted. Among 84 patients resistant to isoniazid and rifampicin 59 were cured, 15 died, two developed treatment failure and eight cases were defaulted. Among 18 patients resistant to isoniazid, rifampicin and ethambutol 14 cured, two died, one developed treatment failure and one defaulted. Among 16 patients resistant to isoniazid, rifampicin and either streptomycin or fluoroquinolone, 11 were cured, two died, one developed treatment failure and two defaulted (shown in Figure 2). There was no statistical difference between these groups with linear by linear association, $p=0.76$.

Of these DRTB cases, 17 were newly diagnosed tuberculosis patients, of which 14 cured, two died, none of them had treatment failure and one case was defaulted. 15 patients had been previously treated with Category 1 regimen of which 11 cured, three died, one patient had failure outcome, and none of the cases were defaulted. 122 patients had been previously treated with category 2 regimen, of which 84 patients were cured, 21 died, five had treatment failure, and 12 defaulted as seen in Figure 3. There was no statistical difference between groups, $p=0.207$ (linear by linear association).

There were 98 male DRTB patients of which 71 cured, 15 died, three patients had failure outcome, and nine cases were defaulted. There were 56 female cases of which 38 cured, 11 died, three had failure, and four of them defaulted. There was no statistical significance between these groups, $p=0.855$ (Pearson Chi square). Of 98 male patients, 21 had resistance to rifampicin, 55 to isoniazid and rifampicin, 12 to isoniazid, rifampicin and ethambutol, and 10 had resistance to isoniazid, rifampicin and either streptomycin or quinolone whereas among 56 female patients, 15 had resistance to rifampicin, 29 to isoniazid and rifampicin, six patient had resistance to isoniazid, rifampicin and ethambutol, and six of them were resistant to isoniazid, rifampicin and either streptomycin or quinolone with $p=0.669$ (linear by linear association).

Among 122 patients previously treated with category 2, 20 had resistance to rifampicin, 68 to isoniazid and rifampicin, 18 to isoniazid, rifampicin and ethambutol, and 16 had resistance to isoniazid, rifampicin and either streptomycin or quinolone. Of 15 patients previously treated with category 1 , eight had resistance to rifampicin, seven cases were resistant to isoniazid and rifampicin, none of them were resistant to ethambutol, streptomycin or quinolone. Of 17 new DRTB patients, eight had resistance to rifampicin, nine cases were resistant to isoniazid and rifampicin, none of them were resistant to ethambutol, streptomycin or quinolone. There was statistical significance between these groups, $p=0.000$, (linear by linear association). Isoniazid, rifampicin and ethambutol combination drug resistance as well as isoniazid, rifampicin along with either streptomycin or ofloxacin drug resistance were only seen in patients previously given Cat 2 ATD, as shown in Fig. 4. No significant difference was seen in treatment outcome with history of tuberculosis treatment (either newly diagnosed or previously treatment with category 1 or category 2 ATD), $p=0.207$, linear by linear association. 


\section{Figure 2: Treatment outcome of DRTB in different resistance groups}

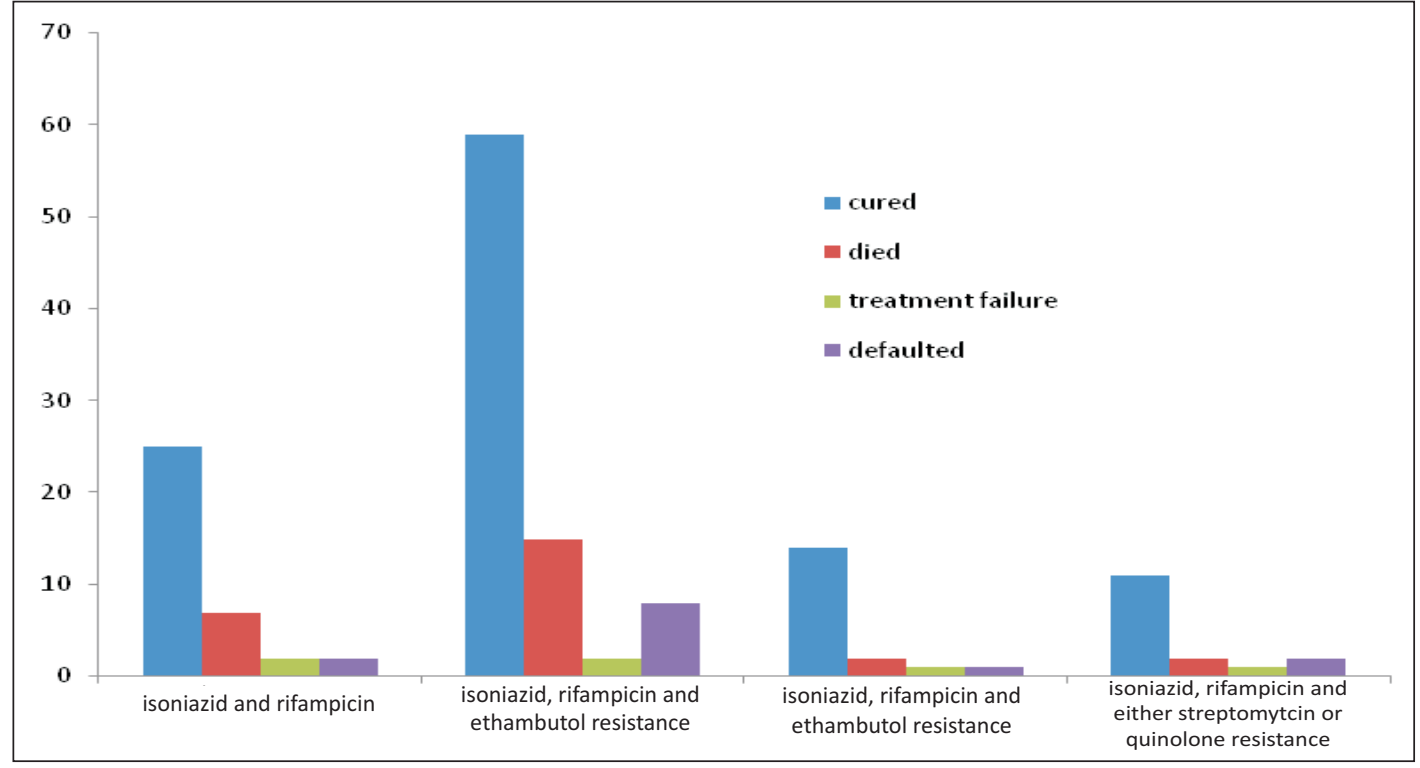

Figure 3: DRTB outcome in different treatment history

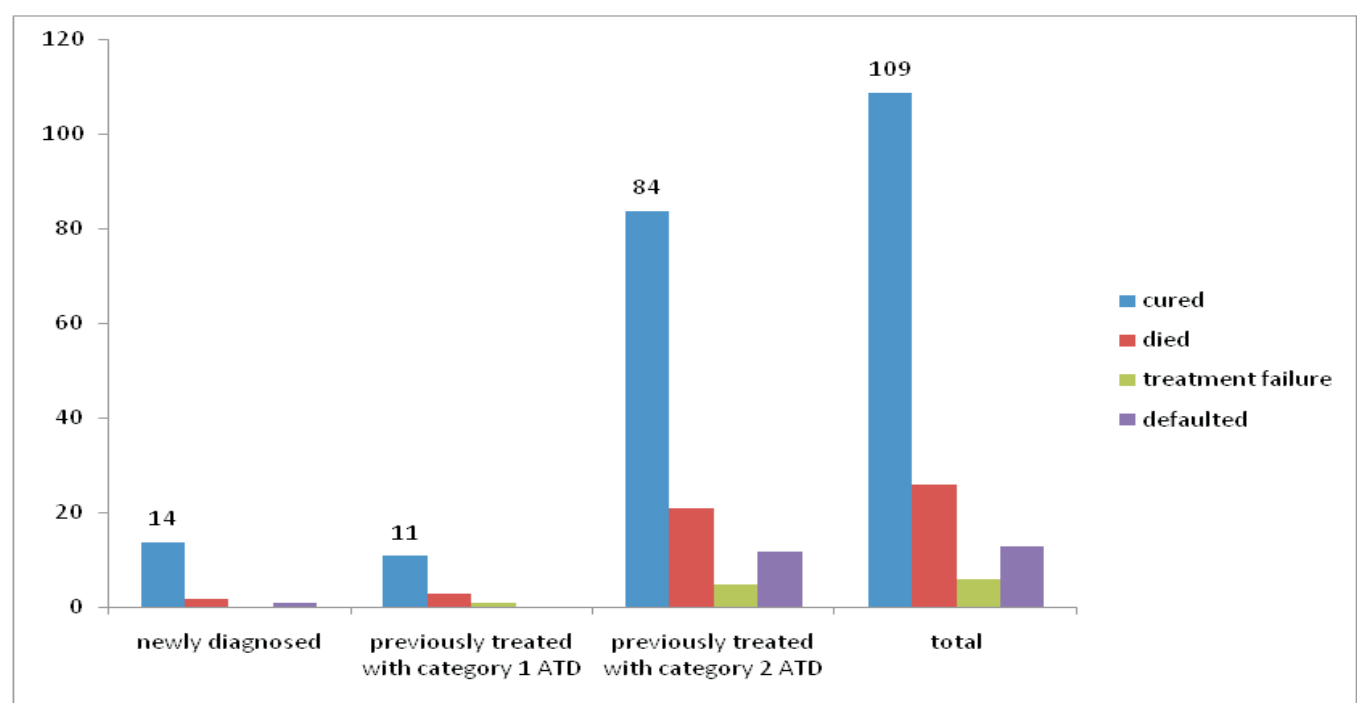

Figure 4: Resistance pattern of DRTB cases according to treatment history

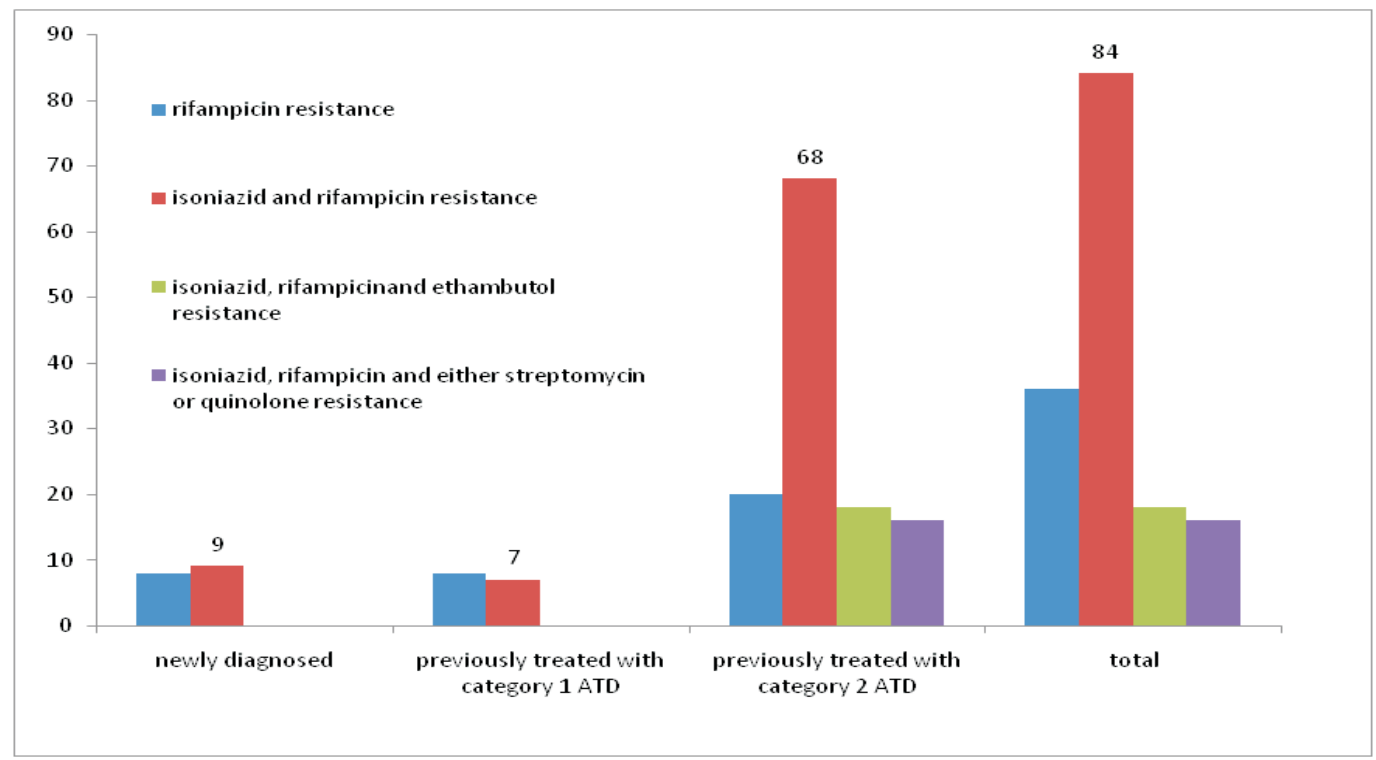


Among these 154 DRTB patients, cases who died were from older age group (mean=41.2 years old, standard deviation=12.85) compared to patients who were cured (mean age $=32.7$ years old, standard deviation=13.53), $p=0.000$, there was no statistical difference between other groups. There was no statistical difference in age as according to the drug resistance pattern $(p=0.10)$. There was also no statistical difference between the different resistance pattern groups with treatment outcome $(p=0.762$, linear by linear association).

Cured patients had earlier sputum smear conversion (mean time=2.1 months, standard deviation=1.71 months, median 2 months) compared with died patients (mean=4.5 months, standard deviation=3.02 months, median 3 months), $p=0.000$. There was no statistical difference in culture conversion period in cured, died, failure and defaulted patients, $p=0.312$.

\section{DISCUSSION}

Our study showed that there was mono as well as multidrug resistance to anti-tuberculosis drugs in the eastern region of Nepal and overall cure rate was around $71 \%$. This result is almost consistent with other recent studies..$^{6,14-16}$ Treatment of drug resistance tuberculosis is under national tuberculosis protocol of Nepal and it follows the guidelines of world health organization and center for disease control and prevention. This may be the reason for worldwide similarity in treatment outcome of drug resistance tuberculosis. Globally treatment success rate of MDRTB is around 50\%, whereas cure rate of drug resistance tuberculosis in this center was $70.78 \%$, death of $16.88 \%$, treatment failure of $3.90 \%$ and default of $8.44 \%$. These numbers seem to be quite satisfactory. However, treatment target rate of $75 \%$ or more for MDRTB was achieved by 43 of 127 countries and territories that reported outcomes in 2012 cohort. In contrary, some 15 years old study showed that cure rates for drug-resistant tuberculosis was variable, highest of about $60 \%$ in Hong Kong to as low as $5 \%$ in Russia, ${ }^{17}$ these may be due to poor recognition and treatment of drug resistant tuberculosis at that period. So, overall treatment outcome of drug resistance tuberculosis from the NATA, Biratnagar seems reasonable with around $71 \%$ cure rate. The reason may be due to use of new technologies like geneXpert for early detection of suspected drug resistance case, directly observed treatment and nutrition as well as other supports provided by the center.

Those patients who were previously treated with category 2 ATD had more number of drug resistant to the Mycobacterium tuberculosis, with ethambutol, streptomycin or quinolone resistance were seen only in patients previously treated with category 2 ATD but not in patients previously treated with category 1 ATD or newly diagnosed drug resistance tuberculosis patients. The result is almost similar to the drug resistance pattern seen in previously treated patients compared to new patients. ${ }^{18-22}$ Improper and/or incomplete treatment can be the main cause of increase rate of drug resistance among previously treated tuberculosis cases compared to new one in Nepalese population. ${ }^{23}$ Previous TB treatment, smoking habit, alcoholism, poverty, poor housing, overcrowding, homelessness, illiteracy and migration from/to high DRTB prevalence areas are other common reasons for the development of drug resistance tuberculosis. ${ }^{24-25}$ It may be due to the fact that when monodrug resistant bacteria are treated first with traditional category 1 and category 2 regimen, there will be higher chances that these bacteria will get resistance to other drugs that were being treated. ${ }^{26}$

Sputum culture with drug sensitivity or molecular tests can detect drug resistance bacteria. Sputum cultures for Myocobacterium tuberculosis and drug sensitivity test require longer duration and to perform requires resource intensive laboratories. GeneXpert on other hand is WHO recommended test that requires minimal training, can be performed rapidly for the detection of tuberculosis bacteria and their sensitivity to rifampicin. Many low and middle income countries qualify for negotiated discounts on this assay. ${ }^{27}$ GeneXpert clinical validation trials ${ }^{13,28-29}$ and operational studies ${ }^{30-31}$ have demonstrated high sensitivity and specificity of this test for both the detection of Mycobacterium tuberculosis and for resistance of rifampicin. It can be recommended that sputum test with GeneXpert/ rifampicin sensitivity may be needed for all those sputum, whose smear shows positivity to acid fast bacilli. In developing countries like Nepal, this may carry higher economy burden than simply having only smear test for acid fast bacilli. But early detection of drug resistance tuberculosis bacteria improves infection control and reduces overall costs. ${ }^{32-33}$ National tuberculosis program of Nepal and world health organization should take this seriously if they want to eradicate or even control DRTB cases as well as overall tuberculosis cases in Nepal.

In our study those patients who died had older age (mean 41.2 years) compared to those who were cured (mean 32.7 years). Similar results were seen in other studies with poor outcome in old aged tuberculosis patients. ${ }^{34-35}$ This may show the decrease in immunity which may be the cause of increase in death among these groups although the mean age of patients who died was only around 40 years. There has been report that more number of drug resistance was seen in older aged tuberculosis patients. ${ }^{26}$ No such findings were seen in our study.

In our study cured patients had earlier sputum smear conversion (mean time=2.1 months, standard deviation=1.71 months, median 2 months) compared with died patients (mean $=4.5$ 
months, standard deviation=3.02 months, median 3 months) with statistical significance. This is pretty consistent with the reports of worse outcome in patients who had delayed sputum conversion rate. ${ }^{36-38}$ Early sputum conversion likely points out the higher probability of cured treatment outcome in DRTB patients. Any delay in period of conversion of sputum smear may alert the likelihood of worse outcome and more intensive measure in treatment may be recommended in these groups of people.

\section{CONCLUSION}

There was overall $71 \%$ cure rate in case of drug resistance tuberculosis. Earlier sputum conversion was seen in cured patients compared to those who died during the treatment. Similarly, more number of drugs was resistant in patients previously treated with category 2 Anti-tubercular drugs compared to other DRTB cases.

\section{LIMITATIONS OF THE STUDY}

This is a single centre study from the data collected in national anti-tuberculosis association, Biratnagar, Nepal and may not represent the overall prevalence and outcome of eastern region of Nepal.

\section{ACKNOWLEDGEMENT}

We are greatly thankful to national anti-tuberculosis association, Biratnagar for providing these valuable data. We are also thankful to all the people who are directly or indirectly involved in this study.

\section{CONFLICT OF INTEREST}

None

11. Van Deun A, Maug AK, Salim MA et al. Short, highly effective, and inexpensive standardized treatment of multidrug-resistant tuberculosis. Am J Respir Crit Care Med. 2010;182(5):684-92.

12. Roadmap for rolling out Xpert MTB/RIF for rapid diagnosis of TB and MDR-TB. World Health Organization; 2010

13. Helb D, Jones M, Story E et al. Rapid detection of Mycobacterium tuberculosis and rifampin resistance by use of on-demand, nearpatient technology. J ClinMicrobiol. 2010;48(1): 229-37

14. Anderson LF, Tamne S, Watson JP et al. Treatment outcome of multidrug resistant tuberculosis in the United Kingdom: retrospective -prospective cohort study from 2004 to 2007. Euro Surveill. 2013;18 (40)

15. Chien JY, Chen YT, Wu SG et al. Treatment Outcome of Patients with Isoniazid Mono-resistant Tuberculosis. ClinMicrobiol Infect. 2015;21(1):59-68.

16. Rao P, Chawla K, Shenoy VP et al. Study of drug resistance in pulmonary tuberculosis cases in south coastal Karnataka. J Epidemiol Glob Health. 2015;5(3) :275-81

17. Raviglioni MC, Dye C, Schmidt S et al. The WHO Global Surveillance and Monitoring Project assessment of worldwide tuberculosis control. Lancet 1997;350:624-9

18. Maurya AK, Kant S, Nag VLet al. Trends of anti-tuberculosis drug resistance pattern in new cases and previously treated cases of extrapulmonary tuberculosis cases in referral hospitals in northern India. J Postgrad Med. 2012;58(3):185-9

19. Shah AR, Agarwal SK, Shah KV. Study of drug resistance in previously treated tuberculosis patients in Gujarat, India. Int J Tuberc Lung Dis. 2002;6(12):1098-101

20. Thapa G, Pant ND, Khatiwada S et al.Drug susceptibility patterns of the Mycobacterium tuberculosis isolated from previously treated and new cases of pulmonary tuberculosis at German-Nepal tuberculosis project laboratory, Kathmandu, Nepal. Antimicrob Resist Infect Control. 2016; 5(1): 30.
10. Guidelines for the programmatic management of drug-resistant tuberculosis. Geneva: World Health Organisation; 2011. 
21. Irfan U, Arshad J, Zarfishan T et al. Pattern of drug resistance and risk factors associated with development of drug resistant Mycobacterium tuberculosis in Pakistan. PLoS One. 2016;11(1): e0147529.

22. Hamusse SD, Teshome D, Hussen MS et al. Primary and secondary antituberculosis drug resistance in Hitossa district of Arsi zone, Oromia regional state, central Ethiopia. BMC Public Health. 2016;16:593.

23. Bhatt $C P$, Bhatt $A B$, Shrestha $B$. Nepalese people's knowledge about tuberculosis. J Tuberc Lung Dis HIV/AIDS. 2009;6(2): 31-37.

24. Marahatta SB, Kaewkungwal J, Ramasoota P et al. Risk factors of Multidrug Resistant Tuberculosis in central Nepal: A pilot study. Kathmandu Univ Med J. 2010;8 (32):392-397. [PubMed]

25. Pant R, Pandey KR, Joshi $M$ et al. Risk Factor Assessment of Multidrug-Resistant Tuberculosis. J Nepal Health Res Counc. 2009;7(15):89-92.

26. Green $\mathrm{E}$, Obi CL, Nchabeleng $\mathrm{M}$ et al. Drug-susceptibility Patterns of Mycobacterium tuberculosis in Mpumalanga Province, South Africa: Possible Guiding Design of Retreatment Regimen. J Health PopulNutr. 2010;28(1):7-13.

27. Negotiated Prices for Xpert MTB/RIF. [http://www. finddiagnostics. org/about/what_we_do/successes/find-negotiatedprices/xpert_mtb_rif.html]

28. Rachow A, Zumla A, Heinrich $\mathrm{N}$ et al. Rapid and accurate detection of Mycobacterium tuberculosis in sputum samples by Cepheid Xpert MTB/RIF assay-a clinical validation study. PLoS ONE 2011; 6(6),e20458.

29. Boehme CC, Nabeta P, Hillemann D et al. Rapid molecular detection of tuberculosis and rifampin resistance. N Engl J Med 2010; 363 (11):1005-15.

30. Steingart KR, Schiller I, Horne DJ et al.Xpert ${ }^{\circledR}$ MTB/RIF assay for pulmonary tuberculosis and rifampicin resistance in adults. Cochrane Database Syst Rev 2014;1,CD009593.
31. Boehme CC, Nicol MP, Nabeta $P$ et al. Feasibility, diagnostic accuracy, and effectiveness of decentralised use of the Xpert MTB/RIF test for diagnosis of tuberculosis and multidrug resistance: a multicentre implementation study. Lancet 2011;377(9776): 1495-505.

32. Van'tHoog $\mathrm{AH}$, Bergval I, Tukvadze $\mathrm{N}$ et al. The potential of multiplex high-throughput molecular assay for early detection of first and secondline tuberculosis drug resistance mutations to improve infection control and reduce costs: a decision analytical modeling study. BMC Infect Dis. 2015;15:473.

33. Tolani MP, D'souza DTB, Mistry NF. Drug resistance mutations and heteroresistance detected using the GenoTypeMTBDRplus assay and their implication for treatment outcomes in patients from Mumbai, India. BMC Infect Dis. 2012;12:9.

34. Ananthakrishnan R, Kumar K, Ganesh Met al. The profile and treatment outcomes of the older (aged 60 years and above) tuberculosis patients in Tamilnadu, South India. PLoS One. 2013;8(7):e67288.

35. Ahmad N, Javiad A, BasitA et al. Management and treatment outcomes of MDR-TB: results from a setting with high rates of drug resistance. Int J Tuberc Lung Dis. 2015;19(9):1109-14,i-ii

36. Joseph P, Desai VB, Mohan NS et al. Outcome of standardized treatment for patients with MDR-TB from Tamil Nadu, India. Indian J Med Res. 2011;133(5):529-34

37. Holtz TH, Sternberg M, Kammerer S et al. Time to sputum conversion in multidrug-resistant tuberculosis: predictors and relationship to treatment. Ann Intern Med. 2006;144 (9):650-9.

38. Kurbatova EV, Cegielski JP, Lienhardt C et al. Sputum culture conversion as a prognostic marker for end-of-treatment outcome in patients with multidrug-resistant tuberculosis: a secondary analysis of data from two observational cohort studies. Lancet Respir Med. 2015;3(3):201-9. 